\title{
A new visual performance test offers new variables for assessing daytime vigilance: Short pauses in performance
}

\author{
TAPIO JOKINEN \\ Helsinki University Central Hospital and KNF-Laboratoriot, Helsinki, Finland \\ OUTI SAARENPÄÄ-HEIKKILÄ \\ Tampere University Hospital, Tampere, Finland \\ and \\ PEKKA LAIPPALA \\ University of Tampere and Tampere University Hospital, Tampere, Finland
}

\begin{abstract}
A new visual performance test, VigiMouse, was evaluated with the aid of 6 volunteering pediatry residents. The results were compared with a visual analogue scale in differentiating four different states: mild sleep deprivation, low blood alcohol level, a combination of both, and the normal state. A normal night shift at a busy pediatric ward was chosen to represent sleep deprivation. A new set of parameters based on short pauses in performance proved to be more sensitive in detecting small changes in performance than parameters based on reaction times.
\end{abstract}

The aim of the visual performance test described in the present article is to detect the performance deficits caused by sleep loss and alcohol and to evaluate the test's usefulness in measuring extensive daytime sleepiness (EDS). Several approaches have been used in testing EDS: analyzing changes of the electroencephalogram (EEG), analyzing performance, and questioning test subjects directly. There are several validated subjective methods for assessing EDS. Hoddes, Zarcone, Smythe, Phillips, and Dement (1973) described a standardized method (Stanford Sleepiness Scale) by validating seven statements describing the subject's vigilance state. Other sets of statements have been validated (e.g., Karolinska Sleepiness Scale; Åkerstedt \& Gillberg, 1990). A similar, simpler test is the visual analogue scale used in this study. The result is given as a single number-distance from the "asleep" point (Hayes \& Pattersson, 1921).

The diagnosis of sleep disorders in clinical practice is mostly based on nighttime polygraphy studies. Carskadon and Dement (1977) suggested that sleep latency, when measured in a consistent and reliable way, could be useful in determining daytime vigilance. This method has several advantages over performance tests. The basic assumption is logical: Apparently, a somnolent person falls asleep faster than a person who is wide awake; also, muscle fatigue, motivation, and learning cannot affect the results. From these basic assumptions, a standardized

A copy of the VigiMouse software can be obtained from the authors on request. Correspondence should be addressed to T. Jokinen, KNFLaboratoriot, Bulevardi 22 A, 00120 Helsinki, Finland (e-mail: tapio@) clinilab.fi). protocol for evaluating sleep latency was developed, the multiple sleep latency test (MSLT; Richardson, Carskadon, Orav, \& Dement, 1978).

However, some patients clinically suffer from EDS, but the MSLT results are normal. It has been suggested that a repeated test of sustained wakefulness (RTSW; Hartse, Roth, \& Zorick, 1982) could be more appropriate for identifying persons with EDS. Both MSLT and RTSW are too tedious and expensive to be used as screening tests.

Roth, Nevsimalova, Sonka, and Docekal (1986) suggested another way of using conventional EEG and polygraphy. A 45-min polygraphic registration is performed, and all latencies and durations of different sleep stages are defined.

There are relatively few standardized performance tests. The continuous performance test (Roswold, Mirsky, Sarason, Bransome, \& Beck, 1956) is based on perceptual sensitivity, as is its adaptation by Nuechterlein, Parasuraman, and Jiang (1983). There are also more recent computerized tests based on visual perception (Gillberg, Kecklund, \& Åkerstedt, 1994). The Mackworth vigilance clock test (Mackworth, 1950), a classical vigilance test in which the subject monitors the hand on a clock for extended periods of time, also exists as a computerized version (Weitkunat \& Bestler, 1990). It has been argued recently that auditory tasks would be more difficult than visual tasks and thus more sensitive to problems in sustained attention (Baker, Taylor, \& Leyva, 1995).

Most vigilance tests based on performance are based on reaction time (RT; e.g., Dinges \& Powell, 1985; Wilkinson, 1970; Wilkinson \& Houghton, 1982). Wilkinson's original RT test lasted for $1 \mathrm{~h}$. The subjects heard a 
0.5 -sec sound with a $2-\mathrm{sec}$ pause - that is, 1,800 sounds during the test. Of these, 40 were a little shorter $(3 / 8 \mathrm{sec})$. The subjects reacted to this variant stimulus by pressing a button. After this, they further chose one out of three options indicating degree of certainty that the stimulus was a variant. The original test was implemented with a tape recorder (Wilkinson, 1970).

Wilkinson later developed his test further and made a commercially available device (Wilkinson \& Houghton, 1982). This device can use either a visual or an auditory stimulus. The test lasts $10 \mathrm{~min}$. The responses are gathered on tape and are later downloaded to a computer for analysis. Another frequently used group of performance tests in recent literature is driving stimulators (e.g., Ingram, Henke, Levin, Ingram, \& Kuna, 1994; Mitler, Hajdukovic, \& Erman, 1993).

In RT tests, the shortest adequate test time depends on the stimulus frequency. For instance, 12 signals in $1 \mathrm{~h}$ is not enough, but 15 signals in $1 \mathrm{~min}$ is enough for a 10 min test (Lisper \& Kjellberg, 1972). Parasuraman and Davies (1976) analyzed the effect of signal probability and event rate and found that both have an effect on correct detections and false alarms, whereas only changes in signal probability have an effect on correct ommissions and omission errors. Nuechterlein et al. (1983) reported a decrease over time in perceptual sensitivity to a visual target when the targets and nontargets were visually highly degraded. These changes were clearly seen after only 5 min of observation.

Many performance tests are primarily constructed for the evaluation of effects of alcohol and drugs (e.g., Korttila, 1988; Nuotto \& Korttila, 1991). Many of these tests include simple RT as one parameter and use a separate test for each additional parameter (e.g., Nuotto \& Korttila, 1991). Rohrbaugh, Stapleton, Parasuraman, and $\mathrm{Zu}-$ bovic (1987) compared the effects of three different alcohol doses on vigilance performance using a visual vig ilance test. They showed a clear interaction between the alcohol dose and not only the decrement but also the rate of vigilance decrement. Their low dose (blood alcohol concentration, $\mathrm{BAC}$, of under $40 \mathrm{mg} \%$ ) did not show a significant difference in hit rates, relative to a placebo.

In the present test, the collection of several parameters was combined into one visual performance test, which we have named VigiMouse (Jokinen, Salmi, Ylikoski, \& Partinen, 1995). We have also introduced new variables (based on pauses during movement) that we propose are more sensitive in detecting the degradation of performance caused by EDS and that have not been previously published. Since we have discussed several classical vigilance tests, it is important to emphasize the distinction between level of vigilance and vigilance decrement. Vigilance level describes the overall level of performance without regard to rate of performance decline (Rohrbaugh, Stapleton, Parasuraman, Frowein, et al., 1987), whereas vigilance decrement concerns performance decline across time. Our interest is specifically in detecting EDS and, thus, in the context of the definition of vigilance, vigilance level. To our knowledge, the way that our test combines a visual RT test with characterizing the motor response to stimuli in detail has not been used before.

We tested VigiMouse on a group of 6 healthy volunteers in sleep deprivation and, in addition, a double-blind administration of a small dose of alcohol. Daytime vigilance was assessed with a visual analogue scale.

\section{METHOD}

\section{Subjects}

Six pediatry residents volunteered for the study. All were women and fulfilled the following criteria: (1) no narcolepsy or obstructive sleep apnea; (2) body mass index (BMI; weight $\times 10,000 /$ length $^{2}$ ) between 18 and 27 (our volunteers, BMI $=18.3-21.1, M=20.7$, $S D=1.9$ ); (3) at least $6 \mathrm{~h}$ of sleep during the 2 nights preceding each test; (4) age under 40 years; (5) not in continuous shift work; and (6) no diseases such as epilepsy, insulin diabetes, asthma, or psychiatric disease.

\section{Testing Procedure}

Each subject performed one set of tests under each of the four different treatment levels. Two sessions were carried out after at least 2 normal nights of sleep, the first without alcohol and the second with alcohol.

Two other sessions were performed after sleep deprivation, again with and without alcohol. Sleep deprivation implied a night on call in a busy pediatric department, where a typical night allows about $1-3$ h of sleep. On the night preceding the tests, the subjects did not sleep at all. The ingestion of coffee or other stimulating substances was forbidden after 4 a.m.

The administration of alcohol was carried out as a double-blind trial. Strongly flavored mixtures were used so that the subjects could not differentiate between alcohol and placebo. The test supervisor did not know which drinks contained alcohol. The order of different treatment sessions for each subject was randomized in order to avoid any serial effects.

The dose of alcohol was $1 \mathrm{~g} / \mathrm{kg}$ of body weight. The aim was to achieve a rather low $0.5-1 \% 00 \mathrm{BAC}$, near $0.5 \%$, which is the maximum permissible BAC for car drivers in Finland; $0.6 \mathrm{dl}$ of $96 \%$ al-

Table 1

Classification and Naming of Test States According to Alcohol Dose and Sleep Deprivation and Means and Standard Deviations of Parameters

\begin{tabular}{|c|c|c|c|c|c|c|}
\hline \multirow[b]{2}{*}{ Alcohol (A) } & \multirow[b]{2}{*}{ Sleep Deprivation (D) } & \multirow[b]{2}{*}{ Test State Name } & \multicolumn{2}{|c|}{ Visual Analogue Scale } & \multicolumn{2}{|c|}{ Breath Alcohol } \\
\hline & & & $M$ & $S D$ & $M$ & $S D$ \\
\hline No & No & $A-D-$ & 17.3 & 6.3 & .017 & .041 \\
\hline No & Yes & $\mathrm{A}-\mathrm{D}+$ & 54.7 & 16.9 & .000 & .000 \\
\hline Yes & No & $A+D-$ & 31.8 & 18.1 & .567 & .082 \\
\hline Yes & Yes & $\mathrm{A}+\mathrm{D}+$ & 69.5 & 18.3 & .733 & .361 \\
\hline
\end{tabular}

Note- $\mathrm{A}-$ indicates no alcohol, and $\mathrm{A}+$ indicates alcohol; $\mathrm{D}-$ indicates no sleep deprivation, and $\mathrm{D}+\mathrm{indi}-$ cates sleep deprivation. 
cohol was added to $4.4 \mathrm{dl}$ of the mixture. BAC was approximated by measuring the breath alcohol level. The different test states are summarized in Table 1.

\section{Visual Analogue Scale}

The visual analogue scale has been used since the $1920 \mathrm{~s}$, when it was first described by Hayes and Pattersson (1921). Current versions of the test utilize a 10 -cm-long line, sometimes with a special movable position marker (Bond \& Lader, 1974; Maxwell, 1978). We used a line of $150 \mathrm{~mm}$. The leftmost end represented "I am falling asleep," and the rightmost end represented "I am wide awake." The subjects indicated their subjective state of vigilance as a mark on the line. The length from that mark to the "wide awake point," calculated in percent of the total length, represents the individual result. Thus, wide awake is $0 \%$, and falling asleep is $100 \%$. The analogue visual scale was applied four times in conjunction with each test during sleep deprivation: at 2200,2400 , and $0400 \mathrm{~h}$, and before the test, at about $1000 \mathrm{~h}$. In the tests performed without sleep deprivation, the analogue scale was applied at $1000 \mathrm{~h}$ before the performance test. The values noted before the test (see Table l) are included in the analysis.

\section{Performance Test}

The performance test (VigiMouse) was performed at $10 \mathrm{a} . \mathrm{m}$. VigiMouse is a computerized, long-lasting performance test. The duration of the test can be selected. In this study, we used a 30-min test.

The stimuli were presented to the subjects on the display screen of an IBM-compatible PC. The target (a $4 \times 4 \mathrm{~cm}$ rectangle with a $1.4 \times 1.4 \mathrm{~cm}$ center $)$ was displayed at random intervals $(10-20 \mathrm{sec})$ randomly in one of eight possible locations on a 34-cm-diameter screen (see Figure 1).

The subject reacted by moving the cursor from the center of the screen to the target using a pointing device (Microsoft Mouse). The characteristics of a Microsoft Mouse have been measured elsewhere (Beringer, 1992; Segalowitz \& Graves, 1990), and it has been found to be suitable for use in RT measurements.

RT was calculated as the time from the appearance of the target on the screen (the stimulus) to the beginning of the movement of the cursor (the subject's initial response). The movement time of the cursor was defined as the time lapse from the subject's initial response to the cursor reaching the target.
The pauses during the movement were calculated as follows. After the subject had moved the mouse as a response to the target and the RT had been calculated, the program continuously checked that the mouse cursor was moving. If the mouse cursor stopped, a timer started to measure the duration of the stop. During the development of the test, we found that pauses under $200 \mathrm{msec}$ in duration are frequent in normal performance; therefore, only pauses lasting longer than $200 \mathrm{msec}$ were registered. The number and duration of pauses during the movement, the number of pauses lasting longer than $10 \mathrm{sec}$, and the velocity of the movement were computed and stored. The duration of the test was also stored.

The test consisted of 100 responses divided into five epochs of 20 responses. The first epoch was omitted from the analysis as a learning phase. After the test, a graphic output of the test results and the following additional parameters were computed, printed, and stored on disk: mean, mode, mean of the 10 best and 10 poorest values (Dinges \& Powell, 1985), the number of responses exceeding $2.5 \times$ mode, and the number of RTs and pauses exceeding $1 \mathrm{sec}$.

The following additional parameters were computed from the RT: the regression coefficient of the responses in Epochs 2-5 (RegRea), the number of RTs under a given limit $(0.1 \mathrm{sec}$; Dinges \& Powell, 1985), the difference between the means of the 10 best and 10 poorest RTs (ReaDelta), and the difference between the means of the 10 best and 10 poorest values for the pauses during movement, movement times, and cursor velocities.

The relationship between RT values and velocity values is described with one parameter (Koherens2; refer to Table 2). An example of the graphical printout from a normal test is given in Figure 2 . Table 2 lists the variables obtained by our test.

\section{Statistical Methods}

Statistical analysis was carried out using an analysis of variance (ANOVA) for repeated measures, in which the repetition was made over the treatment levels. The treatment included four different levels: the set of tests after (1) a normal night without alcohol, (2) a normal night with alcohol, (3) sleep deprivation without alcohol, and (4) sleep deprivation with alcohol. In our study, we were especially interested in the difference between the levels of the treatment. We wanted to find out which parameters detect the different levels. The distributions of the response parameters were skewed, and accordingly, we used the logarithm transformation for the re-
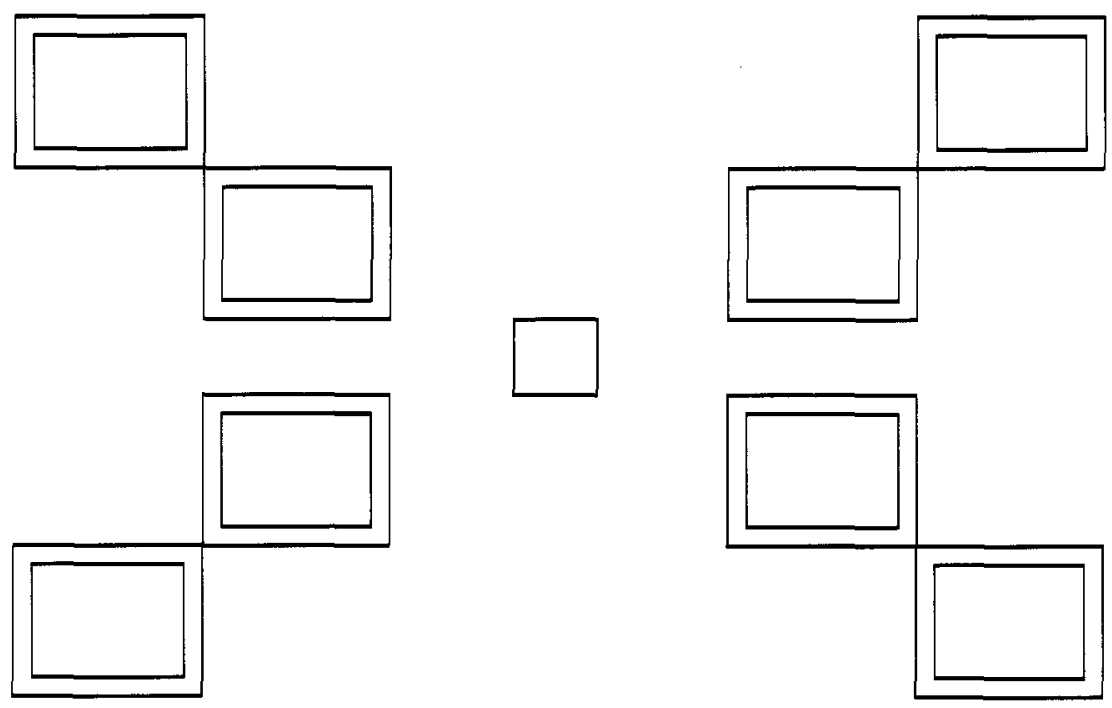

Figure 1. All eight possible target area locations on the screen. Only one of these is randomly selected and shown as a stimulus for each response. 

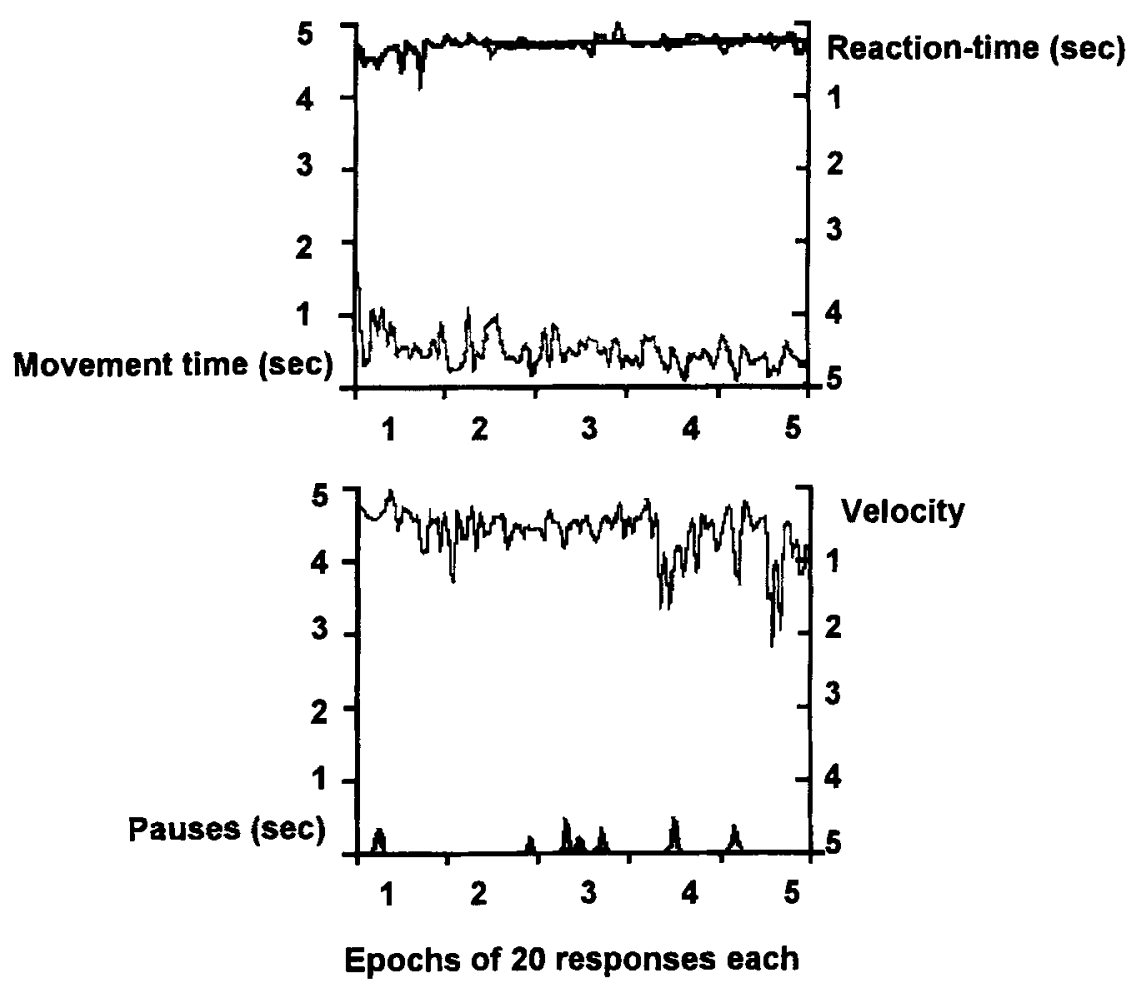

Figure 2. VigiMouse: Sample graphical output, normal subject.

sponse. The sphericity was tested, and the Hyunh-Feldt approximation was applied when necessary. Because the ANOVA result is general, a detailed analysis (post hoc comparison) was carried out using a pairwise $t$ test. In our analysis, we have been intentionally liberal: The limit of significance was set to .10 , and the post hoc comparisons were made without the usually applied correction for mul- tiple comparisons. Our intention was to increase our ability to detect any possible difference, since we could not increase the number of subjects involved. Accordingly, when evaluating our results, one should keep this in mind.

The concordance of the performance test parameters and the visual analogue scale was evaluated using the kappa coefficient to de-

Table 2

Parameters Gathered With VigiMouse

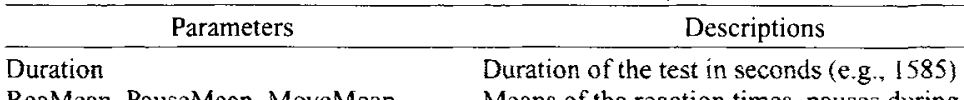

ReaMean, PauseMean, MoveMean, Means of the reaction times, pauses during move-

VeloMean

ment, movement times, and cursor velocities

ReaBest 10, PauseBest10, MoveBest10, Means of the 10 best values

VeloBest 10

ReaWorst 10, PauseWorst 10 ,

MoveWorst 10, VeloWorst 10

ReaDelta, PauseDelta, MoveDelta, VeloDelta

Rea1, Pause 1

Koherens2
Means of the 10 worst values

The difference between the means of 10 best and 10 worst values

Reactions times and pauses of over l sec

Corrected reaction times are calculated as follows:

$\operatorname{Rea}_{\text {corr }}=\frac{\overline{V e l}}{\overline{\text { Rea }}}$ Rea.

The test is divided into epochs of five reactions and means; $\overline{\operatorname{Re} a_{\text {corr }}}$, and $\overline{V e l_{5}}$ are calculated.

Koherens $2=\sum_{1020}\left|R_{\text {earr }}-V e I_{5}\right|$

The regression coefficient of the reaction times Reaction Mode Index. Number of reaction times exceeding $2.5 \times$ mode 
scribe the concordance between two different measuring instruments. When interpreting the kappa values, we regarded agreement with kappa of less than .4 as poor and of .4-.75 as fair to good. Values over .75 indicate very strong agreement (Fleiss, 1981). The kappa coefficients were calculated for a $2 \times 2$ table, which was constructed as follows: First, we produced a $4 \times 4$ table, in which the row variable was the analogue scale score and the column variable was the respective performance test variable. The classification was based on quartiles as cutoff points: below the lower quartile, between the lower quartile and the median, between the median and the upper quartile, and above the upper quartile. The agreement/ disagreement between the analogue scale score and the performance test parameter was defined as shown in Table 3.

In Table 3, "+" denotes agreement, and the final $2 \times 2$ table was constructed on this basis. If the analogue scale and the performance test variable agree, the frequencies are expected to concentrate on the diagonal of the table. The kappa coefficients were calculated only for the parameters for which the quartiles could be defined. We excluded those for which there were too many tied observations.

\section{RESULTS}

The results are summarized in Table 4, which shows the means and, on the basis of the raw data, the standard deviations of the individual performance test parameters in which we were especially interested. The table also includes the ANOVA $p$ values for the treatment levels. Variables based on movement time were not significant in this study; therefore, only the best variable of that group (MoveWorst 10) was included in the table.

The $p$ values were significant for four of seven RTbased performance parameters (ReaWorst 10, ReaMean, Rea1, and ReaDelta). Of the five parameters based on pauses during movement, four had significant $p$ values (PauseWorst 10, PauseMean, PauseDelta, and Pause1). None of the parameters based on movement time and velocities reached significant $p$ values.

The post hoc comparisons and the kappa coefficients indicating the agreement between the respective performance test parameters and the analogue scale are shown in Table 5.

The effect of sleep deprivation alone $(A-D-v s$. $A-D+;$ refer to Table 1) was detected best with the pausederived parameters, which also reached a significant $p$ value in the ANOVA. The respective kappa coefficients showed satisfactory agreement.

Moderate alcohol ingestion ( $A-D-$ vs. $A+D-$ ) was detected with only two parameters: the number of RTs longer than $1 \mathrm{sec}$ (Rea1) and the variable derived from RTs and cursor velocities (Koherens2; refer to Table 2). Rea 1

Table 3

Definition of the Table for Kappa Coefficients

\begin{tabular}{lcccc}
\hline & \multicolumn{4}{c}{ Performance } \\
\cline { 2 - 5 } Analysis & Q1 & Q1-Md & Md-Q3 & Q3 \\
\hline Q1 & + & + & - & - \\
Q1-Md & + & + & + & - \\
Md-Q3 & - & + & + & + \\
Q3 & - & - & + & + \\
\hline
\end{tabular}

Note-“"+" denotes agreement; "--" denotes disagreement. reached a significant ANOVA $p$ value and a satisfactory kappa coefficient, whereas Koherens 2 failed to do so.

As was to be expected, the highest number of detections between states was found in the comparison of the normal state and combined alcohol ingestion and sleep loss $(A-D-v s . A+D+)$, in which four RT-based and three pause-based parameters had significant ANOVA and kappa scores. Pauses longer than $1 \mathrm{sec}$ (Pause 1) had significant $p$ values for the comparison and the overall ANOVA, whereas the kappa coefficient was not satisfactory. Three movement-time-based parameters reached significant $p$ values, although none of these had significant ANOVA values and only the mean of the 10 worst movement times (MoveWorst10) had a satisfactory kappa coefficient.

The addition of alcohol to moderate sleep loss (A-D+ vs. $\mathrm{A}+\mathrm{D}+$ ) was shown by two RT-based and three pausebased parameters, all of which had significant ANOVA and kappa scores.

To summarize the results, the best parameters were based on pauses during movement: PauseWorst 10, PauseMean, and PauseDelta. At a significance level of .10, they detected four, three, and four comparisons, respectively, of the six comparisons. Using a more liberal level of .15, the number of detections was five for each parameter.

Three RT-based parameters-ReaWorst10, ReaDelta, and ReaMean - detected three, two, and two, respectively, of the six comparisons. At a significance level of .15, the number of detections was four for each parameter. Their capacity to detect the respective differences was also confirmed by the agreement with the analogue scale.

\section{DISCUSSION}

Our aim was to create a "real-life" test situation. Instead of the usual type of sleep deprivation, our subjects worked at night as residents in a busy pediatric ward. Their BAC was low, at a level where subjective impairment of performance is not yet accentuated. While there is a difference in the effect of alcohol depending on the time of ingestion (Horne \& Gibbons, 1991), our choice of time was dictated by the test conditions.

According to our results, only one parameter based on RT, the mean of the 10 best RT values (ReaBest), differentiated the normal state from moderate sleep loss $(A-D-$ and $\mathrm{A}-\mathrm{D}+$ states), whereas three parameters based on pauses during movement were statistically significant. The analogue scale, however, was the best single parameter. This suggests that pauses during movement may prove to be a better parameter for detecting the effects of moderate sleep loss than is RT.

This is a logical finding. Let us consider a person who is nearly falling asleep but is trying to react to a stimulus requiring a well-coordinated response. The primary reaction, the first action in the response required, is often swift, but the subject cannot perform the required response appropriately. It has been shown that while alcohol has a minor effect on simple familiar tasks, it has ap- 
Table 4

Means and Standard Deviations of Parameters for Different Treatment Levels

\begin{tabular}{|c|c|c|c|c|c|c|c|c|c|c|}
\hline \multirow[b]{2}{*}{ Variable } & \multirow[b]{2}{*}{ Unit } & \multicolumn{2}{|c|}{$A-D-$} & \multicolumn{2}{|c|}{$\mathrm{A}-\mathrm{D}+$} & \multicolumn{2}{|c|}{$A+D-$} & \multicolumn{2}{|c|}{$\mathrm{A}+\mathrm{D}+$} & \multirow[b]{2}{*}{$p$} \\
\hline & & $M$ & $S D$ & $M$ & $S D$ & $M$ & $S D$ & $M$ & $S D$ & \\
\hline Analogue scale & $\mathrm{mm}$ & 17.33 & 6.28 & 54.67 & 16.91 & 31.83 & 18.06 & 69.5 & 18.23 & $.0001^{*}$ \\
\hline ReaWorst 10 & $\sec$ & .619 & .164 & .718 & .166 & .625 & .087 & .901 & .342 & $.056^{*}$ \\
\hline ReaMean & $\sec$ & .409 & .060 & .444 & .048 & .410 & .028 & .470 & .060 & $.037^{*}$ \\
\hline ReaBest 10 & sec & .270 & .061 & .293 & .055 & .283 & .035 & .294 & .045 & .354 \\
\hline Rea l & $n$ & .167 & .408 & .667 & .816 & 1.000 & .632 & 1.667 & 1.751 & $.082^{*}$ \\
\hline ReaDelta & $\sec$ & .349 & .156 & .425 & .160 & .342 & .103 & .607 & .318 & $.081^{*}$ \\
\hline RMI & & 3.167 & 3.189 & 4.500 & 3.209 & 2.833 & 2.137 & 5.833 & 2.563 & .603 \\
\hline RegRea & & .083 & .085 & .123 & .073 & .045 & .114 & .138 & .227 & .552 \\
\hline Pause Worst 10 & $\sec$ & .136 & .099 & .230 & .103 & .168 & .092 & 1.311 & 2.178 & $.005^{*}$ \\
\hline PauseMean & $\sec$ & .017 & .012 & .030 & .015 & .022 & .015 & .165 & .272 & $.006^{*}$ \\
\hline PauseDelta & $\sec$ & .119 & .086 & .200 & .089 & .145 & .077 & 1.146 & 1.906 & $.005^{*}$ \\
\hline Pause l & $n$ & .000 & .000 & .167 & .408 & .000 & .000 & 1.000 & 1.265 & $.054^{*}$ \\
\hline Move Worst 10 & $\sec$ & .938 & .307 & .949 & .248 & .987 & .205 & 2.052 & 2.455 & .139 \\
\hline
\end{tabular}

Note-A - indicates no alcohol, and A + indicates alcohol; D - indicates no sleep deprivation, and D+ indicates sleep deprivation. Small $p$ values indicate differences between the treatment means. $\quad{ }^{*} p<.10$.

preciable effects in more complicated tasks (Rohrbaugh, Stapleton, Parasuraman, Frowein, et al., 1987). In our test, we did not use false targets. Consequently, RT in our test measured only the less demanding "beginning" of a response, whereas pauses during movement measured the very performance. The pause parameters may be important in assessing the capacity for many important everyday activities, such as the ability to drive a car.

Only one of our parameters - the number of RTs longer than $1 \mathrm{sec}$ (Real) - was able to identify the effect of a small alcohol dose (i.e., the difference between states $A-D-$ and $A+D-)$. Here, the analogue scale had a $p$ value of .12 , which was higher than the $p$ value for sleep loss alone. The alcohol dose used in this study was relatively low, and the breath alcohol levels were only .567 in state $\mathrm{A}+\mathrm{D}-\mathrm{-}$. They were therefore probably too low to be clearly revealed in the level of the test performance.
When comparing the normal state and combined alcohol ingestion and sleep loss $(\mathrm{A}-\mathrm{D}-$ and $\mathrm{A}+\mathrm{D}+)$, the parameters derived from RT and pauses during movement were statistically significant. The RT parameters were more sensitive. The analogue scale was the most sensitive parameter. This again shows that the additional effects of alcohol and sleep deprivation lead to marked impairment of performance with BAC levels near to or lower than the maximum permissible values for car drivers.

Our new parameters, based on pauses during movement, seem to be as reliable as RT-based parameters in assessing clear impairment of performance (in our study, due to combined effect of sleep loss and alcohol), but more sensitive in detecting moderate fatigue.

We conclude that VigiMouse proved to be reliable as an RT-based performance test. Moreover, it offers a new set of parameters based on pauses during movement of the

Table 5

Post Hoc Comparisons of Different Treatment Levels

\begin{tabular}{lccccccccc}
\hline \multicolumn{1}{c}{ Variable } & $\begin{array}{c}\mathrm{A}-\mathrm{D}- \\
\mathrm{A}-\mathrm{D}+\end{array}$ & $\begin{array}{c}\mathrm{A}-\mathrm{D}- \\
\mathrm{A}+\mathrm{D}-\end{array}$ & $\begin{array}{c}\mathrm{A}-\mathrm{D}- \\
\mathrm{A}+\mathrm{D}+\end{array}$ & $\begin{array}{c}\mathrm{A}-\mathrm{D}+ \\
\text { /A+D }\end{array}$ & $\begin{array}{c}\mathrm{A}-\mathrm{D}+ \\
\text { /A+D }+\end{array}$ & $\begin{array}{c}\mathrm{A}+\mathrm{D}- \\
\text { /A+D }+\end{array}$ & $\begin{array}{c}\text { ANOVA } \\
\text { Difference }\end{array}$ & kappa & Concordance \\
\hline Analogue scale & .002 & .067 & .000 & .079 & .002 & .002 & $*$ & & \\
Log Analogue scale & .002 & .123 & .000 & .137 & .241 & .016 & $*$ & & + \\
ReaWorst 10 & .37 & .85 & .09 & .06 & .11 & .04 & $*$ & .62 & + \\
ReaMean & .13 & .87 & .07 & .10 & .21 & .05 & $*$ & .62 & + \\
ReaBest & .17 & .50 & .17 & .58 & .75 & .52 & & .08 & + \\
ReaDelta & .47 & .98 & .11 & .05 & .11 & .03 & $*$ & .75 & ++ \\
Real & .30 & .09 & .10 & .17 & .21 & .40 & $*$ & .46 & + \\
RMI & .93 & .99 & .25 & .14 & .32 & .25 & & .67 & + \\
RegRea & .45 & .51 & .59 & .16 & .88 & .34 & & .42 & + \\
PauseWorst 10 & .04 & .38 & .02 & .12 & .10 & .05 & $*$ & .50 & + \\
PauseMean & .04 & .37 & .02 & .12 & .11 & .05 & $*$ & .50 & + \\
PauseDelta & .04 & .39 & .02 & .12 & .10 & .05 & $*$ & .50 & + \\
Pause1 & .36 & 1.00 & .08 & .36 & .17 & .08 & $*$ & .23 & - \\
MoveWorst10 & .79 & .59 & .13 & .58 & .17 & .26 & & .42 & + \\
\hline
\end{tabular}

Note- $\mathrm{A}$ - indicates no alcohol, and $\mathrm{A}+$ indicates alcohol; $\mathrm{D}-$ indicates no sleep deprivation, and $\mathrm{D}+$ indicates sleep deprivation. " - " indicates an unsatisfactory kappa; "+" indicates a satisfactory kappa; "++" indicates a strong kappa. Exact $p$ values are based on the pairwise $t$ test. ${ }^{*} p<.10$. 
cursor, which proved to be more sensitive in detecting moderate sleep deprivation and low alcohol doses than did parameters based on visual RT.

\section{REFERENCES}

ÅKERSTEDT, T., \& GillberG, M. (1990). Subjective and objective sleepiness in the active indjvidual. International Journal of Neuroscience, 52, 29-37.

Baker, D. B., TAylor, C. J., \& Leyva, C. (1995). Continuous performance tests: A comparison of modalities. Journal of Clinical Psychology, 51, 548-551.

BERINGER, J. (1992). Timing accuracy of mouse response registration on the IBM microcomputer family. Behavior Research Methods, Instruments, \& Computers, 24, 486-490

BOND, A., \& LADER, M. (1974). The use of analogue scales in rating subjective feelings. British Journal of Medical Psychology, 47, 21 1-218.

CarsKadon, M. A., \& Dement, W. C. (1977). Sleep tendency: An objective measure of sleep loss. Sleep Research, 6, 200

DiNGES, D. F., \& POWELL, J. W. (1985). Microcomputer analyses of performance on a portable, simple visual RT task during sustained operations. Behavior Research Methods, Instruments, \& Computers, 17, 652-655

FLEISS, J. (1981). Statistical methods for rates and proportions (2nd ed.). New York: Wiley.

GillberG, M., KeCKLund, G., \& ÅKerstedt, T. (1994). Relations between performance and subjective ratings of sleepiness during a night awake. Sleep, 17, 236-24l.

HARTSE, K. M., Roth. T., \& ZORICK, F. J. (1982). Daytime sleepiness and daytime wakefulness: The effect of instruction. Sleep, 5(Suppl. 2), 107-118.

Hayes, M. H. S., \& PatTersson, D. G. (1921). Experimental development of the graphic rating method. Psychological Bulletin, 18, 98-99.

Hoddes, E., Zarcone, V., Smythe, H., Phillips, R., \& Dement, W. C. (1973). Quantification of sleepiness: A new approach. Psychophysiology, 10, 431-436.

HORNE, J. A., \& GibBons, H. (1991). Effects on vigilance performance and sleepiness of alcohol given in the early afternoon ("post lunch") v5, early morning. Ergonomics, 34, 67-77.

Ingram, F., Henke, K. G., Levin, H. S., Ingram, P. T., \& Kuna, S. T. (1994). Sleep apnea and vigilance performance in a communitydwelling older sample. Sleep, 17, 248-252.

Jokinen, T., Salmi, T., Ylikoski, A., \& Partinen, M. (1995). Use of computerized visual performance test in assessing day-time vigilance in patients with sleep apneas and restless sleep. International Journal of Clinical Monitoring \& Computing, 12, 225-230.

Korttila, K. (1988, January-March). Practical discharge criteria. Problems in Anesthesia, 144-151.

LiSPER, H.-O., \& KJELlberg, A. (1972). Effects of 24-hour sleep de- privation on rate of decrement in a 10 -minute auditory reaction time task. Journal of Experimental Psychology, 96, 287-290.

MACKWORTH, N. H. (1950). Researches on the measurements of human performance (Special Rep. No. 268). London: Medical Research Council.

MAXWELL, C. (1978). Sensitivity and accuracy of the visual analogue scale: A psychophysical classroom experiment. British Journal of Clinical Pharmacology, 6, 15-24.

Mitler, M., Hajdukovic, R., \& Erman, M. K. (1993). Treatment of narcolepsy with methamphetamine. Sleep, 16, 306-317.

Nuechterlein, K. H., Parasuraman, R., \& Jiang, Q. (1983). Visual sustained attention: Image degradation produces rapid sensitivity decrement over time. Science, 220, 327-329.

NuotTo, E., \& KortTILA, K. (1991). Evaluation of a new computerized psychomotor test battery: Effects of alcohol. Pharmacology \& Toxicology, 68, 360-365.

Parasuraman, R., \& Davies, D. R. (1976). Decision theory analysis of response latencies in vigilance. Journal of Experimental Psychology: Human Perception \& Performance, 2, 578-590.

Richardson, G. S., Carsmadon, M. A., Oray, E. J., \& Dement, W. C. (1978). Excessive day-time sleepiness in man: Multiple sleep latency measurements in narcoleptic and control subjects. Electroencephalography \& Clinical Neurophysiology, 45, 621-627.

Rohrbaugh, J., Stapleton, J. M., Parasuraman, R., Frowein, H, ECKART, M. J., \& LiNNoILA, M. (1987). Alcohol intoxication in humans: Effects on vigilance performance. Alcohol \& Alcoholism (Suppl. 1), 97-102.

Rohrbaugh. J., Stapleton, J. M., Parasuraman, R., \& Zubovic, E. A. (1987). Dose-related effects of ethanol on visual sustained attemtion and event-related potentials. Alcohol, 4, 293-300.

Roswold, H. E., Mirsky, A. F., Sarason, I., Bransome, E. D., \& Beck, L. H. (1956). A continuous performance test of brain damage. Journal of Consulting Psychology, 20, 343-350.

Roth, B., Nevsimalova, S., Sonka, K., \& Docekal, P. (1986). An alternative to the MSLT for determining sleepiness in narcolepsy and hypersomnia: Polygraphic score of sleepiness. Sleep, 9, 243-245.

Segalowirz, S. J., \& Graves, R. E. (1990). Suitability of the IBM XT, AT and PS/2 keyboard, mouse and game port as response devices in reaction time paradigms. Behavior Research Methods, Instruments, \& Computers, 22, 283-289

Weitkunat, R., \& Bestler, M. (1990). Computerized Mackworth vigilance clock test. Computer Methods \& Programs in Biomedicine, 32, 147-149.

WILKINSON, R. T. (1970). Methods for research on sleep deprivation and sleep function. International Psychiatric Clinics, 7, 369-381.

Wilkinson, R. T., \& Houghton, D. (1982). Field test of arousal: A portable reaction timer with data storage. Human Factors, 24, 487-493.

(Manuscript received December 13, 1995; revision accepted for publication October $3,1997$. 